\title{
MINIMAL POSITIVE 2-SPANNING SETS OF VECTORS ${ }^{1}$
}

\author{
DANIEL A. MARCUS
}

\begin{abstract}
Let $\left(v_{1}, \ldots, v_{n}\right)$ be a sequence in an $m$-dimensional vector space $V$ over an ordered field such that, for each $i,\left\{0_{j}: j \neq i\right\}$ positively spans $V$. It is shown that if $\left(v_{1}, \ldots, v_{n}\right)$ is minimal with this property, then

$$
n< \begin{cases}4 m & \text { if } m<5 \\ m(m+1) / 2+5 & \text { if } m>5\end{cases}
$$

and all cases are determined in which $n=4 m, m<4$. An application to convex polytopes is given.
\end{abstract}

1. Introduction. Let $V$ be a vector space over an ordered field $K$. A positive spanning set (henceforth abbreviated as PSS) for $V$ is a set $\left\{v_{1}, \ldots, v_{n}\right\}$ of vectors in $V$ such that each vector in $V$ is a linear combination of the $v_{i}$ with nonnegative coefficients. Equivalently, every open half-space in $V$ (one side of a hyperplane) contains some $v_{i}$. This equivalence can be seen by considering the positive span of the $v_{i}$, which is a convex cone with apex at the origin. Either this cone is the entire space or else it lies on one side of a hyperplane.

A positive $k$-spanning set $(\mathrm{P} k \mathrm{SS})$ is a sequence $\left(v_{1}, \ldots, v_{n}\right)$ of vectors in $V$, not necessarily distinct, such that, for each set $I$ of $k-1$ indices $i_{1}, \ldots, i_{k-1},\left\{v_{j}: j \notin\right.$ $I\}$ is a PSS. Equivalently, every open half-space in $V$ contains $v_{i}$ for at least $k$ values of $i$. We are concerned with minimal PkSS's, by which we mean a P $k$ SS such that if any $v_{i}$ is removed, the remaining $v_{j}, j \neq i$, do not form a P $k$ SS.

It is well known that every minimal PSS for $K^{m}$ contains at most $2 m$ vectors; moreover such a set with exactly $2 m$ vectors necessarily consists of a basis for $K^{m}$ and negative multiples of that basis [2, Theorem 6.7]. When $k>1$, it is tempting to conjecture that the "worst case" (the most vectors) occurs when the P $k$ SS consists of $2 \mathrm{~km}$ vectors, $k$ of them in each direction along $m$ linearly independent lines through the origin. Equivalently, the PkSS consists of scalar multiples of some basis.

ConJeCture 1. Every minimal P $k S S$ for $K^{m}$ contains at most $2 k m$ vectors, and every minimal P $k S S$ having exactly $2 \mathrm{~km}$ vectors consists of scalar multiples of some basis for $K^{m}$.

The case $m=1$ is trivial. We will prove

THEOREM 1. Conjecture 1 holds when $m=2$, for all $k>1$.

Received by the editors July 21, 1978.

AMS (MOS) subject classifications (1970). Primary 15A03, 05C99, 52A20.

${ }^{1}$ This research was supported by NSF Grant MCS 78-02526. 
This will be established by a straightforward argument. When $m>3$, however, that argument breaks down at a crucial point. By a more subtle method we will prove the following two theorems, which are the main results of this paper:

THEOREM 2. Conjecture 1 holds when $k=2$ and $m<4$.

TheOREM 3. If $m \geqslant 5$, every P2SS for $K^{m}$ contains at most $m(m+1) / 2+5$ vectors.

Theorems 2 and 3 imply

COROllary 1. When $m<5$, every minimal P2SS for $K^{m}$ contains at most $4 m$ vectors.

Considering the case of two vectors in each direction along $m$ independent lines through the origin, we see that the bound in Corollary 1 is best possible.

2. Positive $k$-spanning sets in combinatorics. There are at least two ways in which positive $k$-spanning sets arise in connection with combinatorial structures. The first (and simpler) of these occurs in the incidence matrix of a digraph (a graph with directed edges), where the element in row $i$ and column $j$ is

$$
a_{i j}=\left\{\begin{array}{cl}
1 & \text { if edge } j \text { terminates at vertex } i \\
-1 & \text { if edge } j \text { originates at vertex } i \\
0 & \text { if otherwise. }
\end{array}\right.
$$

Clearly each column is in the hyperplane $H$ defined by the equation $\Sigma x_{i}=0$. It can be shown [5] that the columns form a PkSS for $H$ if and only if the digraph is $k$-edge-connected. This means that it is connected (there is a directed path from any vertex to any other vertex) and remains so after removal of any $k-1$ edges. Such a digraph is minimally $k$-edge-connected if its $k$-edge connectivity is destroyed by removal of any edge. In this context Conjecture 1 reduces to the statement that every minimally $k$-edge-connected digraph with $m+1$ vertices has at most $2 \mathrm{~km}$ edges, and that if it has exactly $2 \mathrm{~km}$ edges then it can be constructed by replacing each edge of a tree with $2 k$ edges, $k$ in each direction. This special case of Conjecture 1 has been proven by Dalmazzo in [1].

Positive $k$-spanning sets, for $k>2$, also occur in connection with convex polytopes. To each $d$-dimensional convex polytope $P$ with vertices $\boldsymbol{P}_{1}, \ldots, \boldsymbol{P}_{\boldsymbol{n}}$, there is associated a sequence of vectors $\left(v_{1}, \ldots, v_{n}\right)$, called the Gale diagram of $P$, in a real vector space $\mathbf{R}^{m}$ of dimension $m=n-d-1$. The fundamental property of Gale diagrams is this:

Theorem. For each index set $I$, a set $\left\{P_{i}: i \in I\right\}$ is the vertex set of some face $F$ of $P$ if and only if $\left\{v_{i}: i \notin I\right\}$ is a PSS for its linear span, and $F$ is a simplex if and only if $\left\{v_{i}: i \notin I\right\}$ is a PSS for $\mathbf{R}^{m}$. (See [3, \$5.4], or [4] or [6].)

It follows from this (see [3] or [4]) that the Gale diagram of $P$ is always a P2SS for $\mathbf{R}^{m}$, and it is a $\mathrm{P} k \mathrm{SS}$, for $k>3$, if and only if $P$ is $(k-1)$-neighborly. This means that every set of $k-1$ vertices is the vertex set of a face of $P$. Necessarily 
such a face is a simplex. In view of this, Conjecture 1 implies

CONJECTURE 2 . Let $P$ be an $r$-neighborly convex polytope of dimension $d$, and suppose that $P$ has fewer than $(1+1 /(2 r+1))(d+1)$ vertices. Then $P$ contains a vertex $v_{0}$ such that, for each set of $r$ vertices $v_{1}, \ldots, v_{r}$ different from $v_{0}$, the set $\left\{v_{0}, \ldots, v_{r}\right\}$ is the vertex set of a face (necessarily a simplex) of $P$.

The validity of Conjecture 1 for $m<2$ implies that of Conjecture 2 whenever $P$ has at most $d+3$ vertices. When $r=1$, Conjecture 2 reduces to

Conjecture 3. If $P$ is a convex polytope of dimension $d$, and if $P$ has fewer than $4(d+1) / 3$ vertices, then $P$ contains a vertex which is connected to all other vertices of $P$ by edges.

Corollary 1 to Theorems 2 and 3 implies that Conjecture 3 is true for all $d<17$ and also for $d=20$. Whenever $n<d+6$, the number of such vertices is at least $4 d-3 n+4$. Moreover if $n<d+5$ and if $4 d-3 n+4=t$ is the exact number of such vertices, then the Gale diagram of $P$ consists of a minimal P2SS of $4 \mathrm{~m}$ points in $\mathbf{R}^{m}, m<4$, along with $t$ additional points at the origin. Then $P$ is a $t$-fold pyramid on the free join of $n-d-1$ convex quadrilaterals.

3. Proofs of theorems. Before proceeding with the proofs of Theorems 1, 2 and 3, we consider the analog of positive $k$-spanning sets in standard linear algebra. Dropping the assumption that $K$ is an ordered field and the requirement that coefficients be nonnegative, we define a $k$-spanning set of vectors in a $K$-vector space to be a sequence of $n$ vectors, any $n-k+1$ of which form a spanning set for the space. The analog of Conjecture 1 states that every minimal $k$-spanning set for $K^{m}$ contains at most $\mathrm{km}$ vectors, and that a minimal $k$-spanning set with exactly $\mathrm{km}$ vectors consists of scalar multiples of a basis. This can be established by an induction argument as follows:

Obviously the result is true for $k=1$. Proceeding by induction on $k$, assume it holds for minimal $(k-1)$-spanning sets and consider a minimal $k$-spanning set $\left(v_{1}, \ldots, v_{n}\right)$. Assume without loss of generality that $\left(v_{1}, \ldots, v_{n_{0}}\right)$ is a minimal $(k-1)$-spanning set for $K^{m}$. Then $n_{0}<(k-1) m$. We claim that the vectors $v_{n_{0}+1}, \ldots, v_{n}$ are linearly independent. Suppose one of these (say $v_{n}$ ) is a linear combination of the others. Then we will show that $\left(v_{1}, \ldots, v_{n-1}\right)$ is a $k$-spanning set, contrary to assumption. To establish this, consider any set $I$ of $k-1$ indices less than $n$. If at least one of these indices is greater than $n_{0}$, then the $v_{i}, i \notin I$, $i<n_{0}$, form a spanning set for $K^{m}$. Consequently so do all $v_{i}, i \notin I, i<n$. In the other case, if all members of $I$ are less than or equal to $n_{0}$, consider the $v_{i}, i \notin I$, $i<n$. These form a spanning set for $K^{m}$ since all $v_{i}$ form a $k$-spanning set, and their span is the same as that of the $v_{i}, i \notin I, i<n$.

Thus the $v_{i}, i>n_{0}$, are linearly independent. It follows that $n<k m$. Finally, if $n=k m$, then $n_{0}=(k-1) m$ so by inductive hypothesis the $v_{i}, i<n_{0}$, are all scalar multiples of some basis. Also, the $m$ vectors $v_{i}, i>n_{0}$, form a basis. Finally, it is easy to see that these bases must be scalar multiples of each other.

We now consider the extent to which this proof can be adapted to positive $k$-spanning sets over an ordered field. We know Conjecture 1 is true for $k=1$. In the inductive step, we assume that $\left(v_{1}, \ldots, v_{n}\right)$ is a minimal P $k S S$ for $K^{m}$ and that 
$\left(v_{1}, \ldots, v_{n_{0}}\right)$ is a minimal $\mathrm{P}(k-1)$ SS. By the same argument as above, we see that the vectors $v_{n_{0}+1}, \ldots, v_{n}$ are positively independent, which means that none of them is a positive combination of the others. The proof breaks down, however, when we attempt to conclude that $n-n_{0}<2 m$. The problem is that when $m>3$, positively independent sets can be arbitrarily large: Consider, for example, the vertices of a convex $n$-gon in $\mathbf{R}^{3}$, lying in a plane which does not pass through the origin.

When $m=2$, however, a positively independent set can contain no more than four vectors, and if it contains exactly four then they are scalar multiples of a basis. To see this, let $S$ be a positively independent set of vectors in $K^{2}$ and let $u$ and $v$ be two linearly independent vectors in $S$. If all members of $S$ are scalar multiples of $u$ and $v$, then it is easy to see that the assertion is true. If, on the other hand, $S$ contains some vector $w$ which is not a scalar mutiple of $u$ or $v$, then there is a linear dependence $a u+b v+c w=0$, in which $a, b$ and $c$ are all nonzero. If $a, b$ and $c$ are all positive or all negative, then every vector in $K^{2}$ is a positive combination of $u, v$ and $w$, so $S$ contains only three vectors. On the other hand, if $a, b$ and $c$ are not all of the same sign, then one of the vectors $u, v, w$ is a positive combination of the other two, contrary to the assumption that $S$ is positively independent.

We have proved Theorem 1.

Proof of Theorem 2. Theorem 2 holds for $m=1$ trivially. Proceeding by induction on $m$, fix $m=2,3$ or 4 and assume the result for all minimal positive 2-spanning sets in dimension $m-1$. Let $\left(v_{1}, \ldots, v_{n}\right)$ be a minimal P2SS for $K^{m}$. Note that for each $i$, there exists some $j \neq i$ such that $v_{i}$ and $v_{j}$ are alone in some open half-space (i.e., no $v_{k}, k \neq i, j$, is in this open half-space). If this were not true, then deletion of $v_{i}$ would yield a smaller P2SS.

At this point we split the proof into two cases.

Easy case. Suppose two of the $v_{i}$ (say $v_{1}$ and $v_{2}$ ) are on the same ray from the origin. Equivalently, they are positive multiples of each other. Then there exists a hyperplane $H$, one side of which contains no $v_{i}, i>3$. Factoring out the subspace spanned by $v_{1}$, we project all $v_{i}, i>3$, on $H$. Let $v_{i}$ go to $h_{i} \in H .\left(h_{3}, \ldots, h_{n}\right)$ is a P2SS for $H$, so at most $4(m-1)$ of the $h_{i}$ form a minimal P2SS for $H$. Consider the corresponding $v_{i}$ : If at least two of these are not in $H$, then these $v_{i}$ (at most $4(m-1)$ vectors) along with $v_{1}$ and $v_{2}$ form a P2SS. In any case, at most two other $v_{i}$ need be added to this set so that at least two $v_{i}$ are on the opposite side of $H$ from $v_{1}$, and this results in a P2SS containing at most $4 m$ vectors. Moreover the characterization of a minimal P2SS with $4(m-1)$ vectors in $H$ leads to the corresponding result in $\mathbf{R}^{m}$ : Assuming that $n=4 m$, it must be the case that exactly $4(m-1)$ of the $h_{i}$ (say all $\left.h_{i}, i>4\right)$ form a minimal P2SS for $H$, in which case they must all be scalar multiples of a basis for $H$. Moreover none of the $v_{i}, i>4$, can be on the opposite side of $H$ from $v_{1}$, since $v_{3}$ and $v_{4}$ are both necessary for a P2SS for $\mathbf{R}^{m}$. It follows that $v_{i}=h_{i}$ for all $i>4$, and it remains only to show that $v_{3}$ and $v_{4}$ are both scalar multiples of $v_{1}$. Equivalently, $h_{3}$ and $h_{4}$ are both 0 . But this is clear, since for example if $h_{3} \neq 0$, then (in view of the special nature of the $h_{i}, i>4$ ) $h_{3}$ could have been chosen as part of a minimal P2SS for $H$ in the argument above, leading to a P2SS for $\mathbf{R}^{m}$ with fewer than $4 m$ vectors. 
That completes the induction step in the easy case.

Hard case. Suppose no two of the $v_{i}$ are on the same ray from the origin. We introduce a graph $G$ having vertices $1, \ldots, n$, in which $\{i, j\}$ is an edge if $v_{i}$ and $v_{j}$ appear alone in some open half-space. Define, for each $i, A_{i}=\{j:\{i, j\}$ is an edge\}. For any $i$, we change the original P2SS as follows:

remove $v_{i}$;

"double" all vectors $v_{j}, j \in A_{i}$; that is, introduce another copy of $v_{j}$.

Clearly, we still have a P2SS; reduce it to a minimal P2SS. It is easy to see that if a vector $v_{k}$ is removed, and if $j \in A_{k}$, then $v_{j}$ must have been one of the vectors that was doubled. Consequently $j \in A_{i}$. In other words, $k$ is in the set $B_{i}=\left\{k: A_{k} \subseteq\right.$ $A_{i}$ \}. Note also that $i \in B_{i}$ trivially. Thus a new minimal P2SS has been formed by doubling all $v_{i}, i \in A_{i}$, and removing certain of the $v_{k}, k \in B_{i}$ (although not necessarily all such $v_{k}$ ).

Suppose $\left|B_{i}\right|<\left|A_{i}\right|$. Then the new minimal P2SS contains at least $n$ vectors, which are not all distinct. By the easy case, we conclude that $n<4 m$.

Thus if $G$ contains a vertex $i$ such that $\left|B_{i}\right|<\left|A_{i}\right|$, we are finished. We will show that this is the case if $m<3$, and that if $m=4$ all "bad" graphs can be determined and dealt with by another argument.

LEMMA 1. For each $i$, the $v_{j}, j \in A_{i}$, are linearly independent.

Proof. $v_{i}$ is a positive combination of the $v_{k}, k \neq i$; hence by a well-known argument of Carathéodory (see [3, Theorem 2.3.5]) it follows that $v_{i}$ is a positive combination of a linearly independent subset of $\left\{v_{k}: k \neq i\right\}$. Clearly all $v_{j}, j \in A_{i}$, must be in this subset.

Thus each vertex of $G$ has degree at most $m$. Moreover from the definition of $G$ it is clear that $G$ contains no isolated vertices (vertices of degree 0 ). Using only these properties, we can consolidate most of the remainder of the proof into a strictly graph-theoretic lemma:

LEMMA 2. Let $G$ be a graph with no isolated vertices and let $d$ be the maximum degree of all vertices in $G$. If $d<3$, then $G$ contains a vertex $i$ for which $\left|B_{i}\right|<\left|A_{i}\right|$. If $d=4$ and $\left|B_{i}\right|>\left|A_{i}\right|$ for each vertex $i$, then each connected component of $G$ consists of a circuit with two extra edges at each vertex:

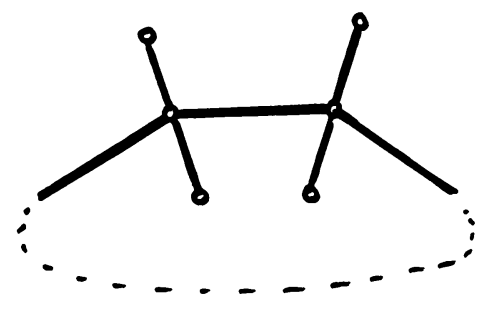

Before proceeding with the proof of Lemma 2, we establish another lemma:

Lemma 3. If $A_{j} \subseteq B_{i}$, then $B_{j} \subseteq A_{i}$. 
Proof. If $b \in B_{j}$, then $A_{b} \subseteq A_{j} \subseteq B_{i}$. Fix any $a \in A_{b}$ ( $G$ contains no isolated vertices); then $b \in A_{a} \subseteq A_{i}$.

Proof of Lemma 2. Assume that $\left|B_{i}\right|>\left|A_{i}\right|$ for each vertex $i$. First we show that $d>4$. Fix a vertex $i$ of degree $d$. Let $A_{i}=\left\{a_{1}, \ldots, a_{d}\right\}, B_{i}=\left\{b_{1}, \ldots, b_{e}=i\right\}$, and let $d_{1}, \ldots, d_{e}$ denote the degrees of $b_{1}, \ldots, b_{e}$. We can assume $d_{1}<d_{2}$ $<\cdots<d_{e}=d$. For each $b \in B_{b_{1}}$, we have $A_{b} \subseteq A_{b_{1}} \subseteq A_{i}$; hence $b \in B_{i}$. Then $b=b_{k}$ for some $k$. Moreover $\left|A_{b_{k}}\right|=d_{k}>d_{1}=\left|A_{b_{1}}\right|$, implying that $A_{b_{k}}=A_{b_{1}}$. Thus without loss of generality we can assume that $A_{b_{1}}=A_{b_{2}}=\cdots=A_{b_{r}}$, where $r=\left|B_{b_{1}}\right|>d_{1}$. Then any $j \in A_{b_{1}}$ is adjacent to $b_{1}, \ldots, b_{r}$, implying $r<d$. It follows that $r<e$; hence $i \neq b_{1}, \ldots, b_{r}$. But every $j \in A_{b_{1}}$ is adjacent to $i$, so in fact $r<d$. Moreover, if $r=d-1$, then $A_{j}=\left\{b_{1}, \ldots, b_{r}, i\right\} \subseteq B_{i}$; hence by Lemma $3, B_{j} \subseteq A_{i}$. But then $\left|B_{j}\right|<\left|A_{i}\right|=d=r+1=\left|A_{j}\right|$, contrary to assumption. Thus we have $1<d_{1}<r<d-2$, implying that $d>4$.

Now assume that $d=4$. Continuing the argument above, we must have $d_{1}=1$ and $r=2$. We claim that of the four vertices adjacent to $i$, two have degree 1 and two have degree 4.

Let $A_{b_{1}}=A_{b_{2}}=\{a\}$. We can assume $a=a_{1}$.

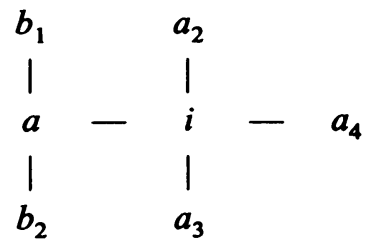

If $a$ has degree 3, then $A_{a} \subseteq B_{i}$; hence by Lemma 3, $B_{a} \subseteq A_{i}$. Since $\left|B_{a}\right|>\left|A_{a}\right|=$ 3, we have $B_{a}=A_{i}$. Again by Lemma 3, $B_{i} \subseteq A_{a}$. But then $\left|B_{i}\right|<\left|A_{i}\right|$. We conclude that $a$ has degree 4 .

Let $A_{a}=\left\{b_{1}, b_{2}, i, j\right\} .\left|A_{j}\right|<4$ and $\left|B_{a}\right|>\left|A_{a}\right|=4$, so $B_{a}-A_{j}$ contains some $k$. $A_{k} \subseteq A_{a}$, but $j \notin A_{k}$. In particular, $k \neq a$, so $b_{1}, b_{2} \notin A_{k}$. Thus $A_{k}=\{i\}$ and we can assume that $k=a_{2}$. $k$ has degree 1 , so $\left|B_{k}\right|>2$. Each member of $B_{k}$ is adjacent only to $i$, so we can assume that $a_{3}$ has degree 1 .

It remains to show that $a_{4}$ has degree $4 .\left|B_{i}\right|>\left|A_{i}\right|=4=\left|A_{a}\right|$, so $B_{i}-A_{a}$ contains some $b$. Necessarily $A_{b}=\left\{a_{4}\right\}$. The argument above can then be repeated with $b_{1}$ replaced by $b$, yielding the fact that $a_{4}$ has degree 4 .

Thus, as claimed, $i$ is adjacent to two vertices of degree 1 and two vertices of degree 4. Since $i$ was an arbitrary vertex of degree 4, we conclude that every vertex of degree 4 is adjacent to two vertices of degree 1 and two vertices of degree 4. Moreover the argument shows that each connected component of $G$ contains a vertex of degree 4, which is the maximum degree in $G$. (Otherwise, by applying the argument to a single component having no vertex of degree 4 , we would obtain a contradiction.) Finally, then, it follows that each component consists of a circuit with two extra edges at each vertex, as claimed. Lemma 2 is now proved.

We now resume the proof of Theorem 2. Lemma 1 shows that the maximum degree $d$ of vertices in $G$ is at most $m$, which is at most 4 . Thus, by Lemma $2, G$ contains a vertex $i$ for which $\left|B_{i}\right|<\left|A_{i}\right|$ except in the case in which each component of $G$ consists of a circuit with two extra edges at each vertex. In that case, 
either $G$ contains at most 15 vertices, or else $G$ contains two nonadjacent vertices $i, j$ of degree 4 such that $A_{i}$ and $A_{j}$ are disjoint. In the latter case, consider the set $\left\{v_{k}: k \neq i, j\right\}$. This is a PSS for $K^{4}$; hence it contains a minimal PSS. The latter necessarily contains $A_{i} \cup A_{j}$. A minimal PSS with at least eight vectors consists of a basis for $K^{4}$ and negative multiples of that basis; hence $\left\{v_{1}, \ldots, v_{n}\right\}$ contains eight such vectors. It then follows (see Lemma 4) that $n<16$, and that $n=16$ if and only if all $v_{i}$ are scalar multiples of the basis.

LEMMA 4. Let $\left(v_{1}, \ldots, v_{n}\right)$ be a minimal P2SS for $K^{m}$ containing a basis for $K^{m}$ and negative multiples of that basis. Then $n<4 m$, and if $n=4 m$ then all $v_{i}$ are scalar multiples of that basis.

Proof. Without loss of generality we can assume that the basis is $\left(v_{1}, \ldots, v_{m}\right)$ and that $v_{m+1}, \ldots, v_{2 m}$ are negative multiples of this basis. For each $i>2 m$ let $v_{i}=a_{i 1} v_{1}+\cdots+a_{i m} v_{m}$. Since $\left(v_{1}, \ldots, v_{n}\right)$ is a P2SS, for each $j=1, \ldots, m$ there must exist $i>2 m$ such that $a_{i j}>0$ and $i>2 m$ such that $a_{i j}<0$. Selecting two such values of $i$ for each $j$, and including $v_{1}, \ldots, v_{2 m}$, we obtain a P2SS consisting of at most $4 m$ vectors. Thus $n<4 m$. If any $v_{i}$ is not a scalar multiple of one of the basis vectors, then it can be chosen for more than one value of $j$, and consequently the P2SS contains fewer than $4 m$ vectors.

Proof of Theorem 3. The proof of Theorem 3 is accomplished by induction on $m$, using an adaptation of the argument in the proof of Theorem 2. Starting with $m=5$ and assuming that $\left(v_{1}, \ldots, v_{n}\right)$ is a minimal P2SS for $K^{5}$, we consider the same two cases as in Theorem 2:

Easy case. If two $v_{i}$ are on the same ray from the origin, then the argument employed in the easy case of Theorem 2, along with the result of Theorem 2 for $m=4$, implies that $n<20$. Moreover it shows that if $n=20$ and two $v_{i}$ are on the same ray from the origin, then all $v_{i}$ are scalar multiples of some basis.

$H$ ard case. If no two $v_{i}$ are on the same ray from the origin, form the graph $G$ as in the proof of Theorem 2. Define $A_{i}$ and $B_{i}$ for each $i$ as before. For any $i$, change the P2SS as before. Suppose, for some $i$, we have $\left|B_{i}\right|<\left|A_{i}\right|+1$. Then the new minimal P2SS contains at least $n-1$ vectors. But the vectors of this set are not all distinct, so by the easy case we have $n-1<20$; moreover if $n-1=20$, then the new set consists of scalar multiples of some basis. In this latter case, we conclude that the original P2SS $\left(v_{1}, \ldots, v_{n}\right)$ contains a basis and negative multiples of that basis. But then, by Lemma $4, n<20$.

Thus in all cases, $n \leqslant 20$ when $m=5$, provided that $G$ contains a vertex $i$ for which $\left|B_{i}\right|<\left|A_{i}\right|+1$. We will show that such a vertex exists, except in one special case which will be handled separately. More generally, we will establish

LEMMA 5. Let $G$ be a graph with no isolated vertices and let $d$ be the maximum degree of all vertices in $G$. If $d>4$, then either $G$ contains a vertex $i$ for which $\left|B_{i}\right|<\left|A_{i}\right|+d-4$, or else each connected component of $G$ consists of a circuit with $d-2$ extra edges at each vertex. 
Proof. Assume that $\left|B_{i}\right|>\left|A_{i}\right|+d-4$ for each $i$. As in the proof of Lemma 2, fix a vertex $i$ of degree $d$ and set $A_{i}=\left\{a_{1}, \ldots, a_{d}\right\}, B_{i}=\left\{b_{1}, \ldots, b_{e}=i\right\}$. As in the proof of Lemma 2, we can assume that $A_{b_{1}}=A_{b_{2}}=\cdots=A_{b_{r}}$ where $r=\left|B_{b_{1}}\right|$. By the same argument as in Lemma 2, we obtain $r<d-2$. Moreover we have $r=\left|B_{b_{1}}\right|>\left|A_{b_{1}}\right|+d-4=d_{1}+d-4$ where $d_{1}$ is the degree of $b_{1}$. Since $d_{1}>1$, we obtain $r>d-2$. Thus $r=d-2$, and it follows that $d_{1}=1$.

We claim that $i$ is adjacent to two vertices of degree $d$, and to $d-2$ vertices of degree 1.

Let $A_{b_{1}}=\cdots=A_{b_{r}}=\{a\}$ and without loss of generality assume $a=a_{1}$. The set $A_{a}$ includes $i, b_{1}, \ldots, b_{r}$ and at most one other vertex, since $r=d-2$. If no other vertex is adjacent to $a$, then a contradiction results as in the proof of Theorem 2. (The only change here is that $\left|A_{a}\right|=d-1$.) We conclude that $A_{a}=\left\{b_{1}, \ldots, b_{r}, i, j\right\}$ for some vertex $j$. Thus $a=a_{1}$ has degree $d$.

As in the proof of Theorem $2, B_{a}-A_{j}$ contains some vertex $k$, which can be shown to be adjacent only to $i$. Moreover the same is true for all members of $B_{k}$, of which there must be at least $\left|A_{k}\right|+d-3=d-2$. Thus, without loss of generality, we can assume that $a_{2}, a_{3}, \ldots, a_{d-1}$ all have degree 1 .

It remains to show that $a_{d}$ has degree $d$. We have $\left|B_{i}\right|>\left|A_{i}\right|+d-4=2 d-4$ $>d>\left|A_{a}\right|$, so $B_{i}-A_{a}$ contains some $b$. Necessarily $A_{b}=\left\{a_{d}\right\}$. The argument above can then be repeated with $b_{1}$ replaced by $b$, yielding the fact that $a_{d}$ has degree $d$.

Thus $i$, an arbitrary vertex of degree $d$, is adjacent to two vertices of degree $d$ and to $d-2$ vertices of degree 1 . As in the proof of Lemma 2, the characterization of $G$ then follows easily. That completes the proof of Lemma 5.

Returning to the proof of Theorem 3, we apply Lemma 5 to complete the case $m=5$, except when each component of $G$ consists of a circuit with $d-2$ extra edges at each vertex. If $d<5$, then $\left|B_{i}\right|<\left|A_{i}\right|+1$ for each $i$, so the only remaining case that must be considered is when $d=5$. In that case, either $n<20$ or else $G$ contains nonadjacent vertices $i$ and $j$ of degree 5 such that $A_{i}$ and $A_{j}$ are disjoint. In the latter case, the proof is completed as in Theorem 2, using Lemma 4.

Finally, we establish the induction step when $m>5$. Lemma 5 implies that either $G$ contains a vertex $i$ for which $\left|B_{i}\right|<\left|A_{i}\right|+m-4$, or else each component consists of a circuit with $m-2$ extra edges at each vertex. In the former case, the vertex $i$ can be used for the induction step. In the latter case, either $n<5(m-1)$ $<m(m+1) / 2+5$, or else $G$ contains nonadjacent vertices $i$ and $j$ of degree $m$ with $A_{i}$ and $A_{j}$ disjoint, and the proof is completed as before.

\section{REFERENCES}

1. M. Dalmazzo, Nombre d'arcs dans les graphes $k$-arc-fortement connexes minimaux, C. R. Acad. Sci. Paris Sér. A-B 285 (1977), 341-344.

2. C. Davis, Theory of positive linear dependence, Amer. J. Math. 76 (1954), 733-746.

3. B. Grünbaum, Convex polytopes, Interscience, New York, 1967.

4. D. Marcus, Gale diagrams of convex polytopes and pasitive spanning sets of vectors (to appear).

5. Circulation polytopes associated with directed graphs (to appear).

6. P. McMullen, Representations and diagrams (to appear).

Department of Mathematics, California State Polytechnic University, Pomona, California 91768 